Chapman University

Chapman University Digital Commons

Pharmacy Faculty Articles and Research

School of Pharmacy

$10-13-2014$

\title{
Concordance of Pharmacist Assessment of Medication Nonadherence With a Self-Report Medication Adherence Scale
}

\author{
Michael S. Kelly \\ Chapman University, mkelly@chapman.edu \\ Leticia R. Moczygemba \\ Virginia Commonwealth University \\ Sharon S. Gatewood \\ Virginia Commonwealth University
}

Follow this and additional works at: http://digitalcommons.chapman.edu/pharmacy_articles

\section{Recommended Citation}

Kelly MS, Moczygemba LR, Gatewood SS. Concordance of pharmacist assessment of medication nonadherence with a self-report medication adherence scale. J Pharm Pract. 2014;29(3):194-198. doi: 10.1177/0897190014550003

This Article is brought to you for free and open access by the School of Pharmacy at Chapman University Digital Commons. It has been accepted for inclusion in Pharmacy Faculty Articles and Research by an authorized administrator of Chapman University Digital Commons. For more information, please contact laughtin@chapman.edu. 


\section{Concordance of Pharmacist Assessment of Medication Nonadherence With a Self-Report Medication Adherence Scale}

\section{Comments}

This is a pre-copy-editing, author-produced PDF of an article accepted for publication in Journal of Pharmacy Practice, volume 29, issue 3, in 2014 following peer review. The definitive publisher-authenticated version is available online at DOI: 10.1177/0897190014550003.

\section{Copyright}

The authors 
Concordance of pharmacist assessment with self-report scales to detect medication nonadherence during a medication review in homeless patients served at a mental-health clinic.

Keywords: medication adherence, medication therapy review, pharmacist, self-report scale 


\begin{abstract}
Objective. To evaluate the concordance of the Modified Morisky Medication Adherence Scale (MMAS) with a pharmacist assessment of medication adherence during a medication review in a group of patients served by a homeless behavioral health clinic.

Methods. This was a retrospective study that examined the electronic medical records (EMR) of patients $\geq 18$ years with a mental health and/or substance abuse diagnosis who received at least 1 medication review by a pharmacist from October 2008 to September 2009 at a homeless behavioral health clinic in Virginia. Patients had to have recorded responses to at least the first 4 questions of the 6-item MMAS to be eligible for the study. The MMAS includes the following yes/no questions: Do you ever forget to take your medicine? Are you careless at times about taking your medicine? When you feel better do you sometimes stop taking your medicine? Sometimes if you feel worse when you take your medicine, do you stop taking it? Do you know the long-term benefits of taking your medicine? Sometimes do you forget to refill your prescription medicine on time? Pharmacists administered and recorded patient responses to the MMAS at the beginning of the medication review. Responses were dichotomized into 2 categories, adherent or non-adherent. In addition to the 6-item MMAS, adherence was calculated using the first 4 and 5 questions of the MMAS due to concerns about over or underestimation of non-adherence based upon whether or not patients were aware of the long-term benefits of medications or did not forget to pick up refills, but did not have transportation to do so. The pharmacist further assessed medication adherence by asking probing questions about responses to the MMAS questions. A final pharmacist assessment of adherence based upon these questions and information gathered during the medication review was documented in the EMR. Sociodemographic information was also collected. Descriptive statistics were calculated for all variables. The kappa statistic was used to assess the agreement between the variations of the MMAS (6, 5, and 4 items) and the pharmacist assessment of medication adherence.
\end{abstract}

Results. A total of 288 patients were eligible for the study, which included 449 medication reviews. The sample was 51.7\% women, 60.4\% African American, and had a mean $( \pm \mathrm{SD})$ age of $44.8( \pm 10.1)$ years. The majority (59.0\%) received two or more medication reviews. Non-adherence was identified in $61.7 \%$, $54.9 \%$, and $49.7 \%$ of medication reviews using the 6,5 , and 4 -item scales, respectively. The pharmacist assessment determined non-adherence in $23.8 \%$ of medication reviews. Fair agreement was observed between the 5 and 4-item scales (kappa statistic $=0.21$ and 0.23 , respectively) and the pharmacist adherence assessment, whereas there was weak agreement between the 6-items and pharmacist assessment $($ kappa statistic $=0.17)$.

Conclusion. The low agreement between the MMAS (6, 5, and 4-items) was unexpected and suggests that the MMAS is not a good fit for assessing medication adherence in this population. For this population, a combination of methods including self-report, pharmacist assessment and refill records, when available, may provide the greatest insight into adherence. 


\section{BACKGROUND}

Medication adherence is defined as the extent to which patients take medications as prescribed by their health care providers. ${ }^{1}$ In the United States, it is estimated that one-third to one-half of patients do not adhere to medications as prescribed by their physician. ${ }^{2}$ Non-adherence to medications can lead to an increased risk of hospitalizations, where an estimated one-third of hospital admissions related to adverse drug events were due to non-adherence. ${ }^{3}$ Further, medication-related morbidity, including non-adherence, is reportedly responsible for up to $\$ 290$ billion (13\%) of the total U.S annual health care expenditures. ${ }^{2}$

Several methods to measure adherence exist, yet there is no consensus on a "gold-standard" method. Direct measures, such as measuring biologic fluid, help ensure medication is ingested, but can be expensive, labor-intensive, and invasive. ${ }^{4}$ Indirect measures (pill counts, prescription refill data, and electronic monitoring) are less invasive to the patient, but can be costly, time-consuming, and inaccurate. ${ }^{4}$ Self-report questionnaires, are an inexpensive and quick way to measure a patients' adherence, but may overestimate adherence due to patient recall and social desirability bias. ${ }^{4}$ Multiple self-report adherence scales have been developed, including the Brief Medication Questionnaire (BMQ), Medication Adherence Rating Scale (MARS), and Morisky Medication Adherence Scale (MAS). ${ }^{5}$ Each self-report scale differs in its sensitivity and specificity, ease of clinical use and scoring, and validity among disease states. A recent literature analysis of 5 self-report adherence scales found the Morisky MAS to be the quickest to administer and simple for a clinician to score. ${ }^{5}$ Further, the MAS has been validated in patients with hypertension, type 2 diabetes, dyslipidemia, and depression as a way to assess adherence. ${ }^{5-10}$ Despite their relative ease of use, self-report methods have not shown consistently high agreement with other indirect measures of adherence. Several studies have reported on the concordance between indirect adherence methods (pharmacy refill data, pill counts, and electronic monitoring) and self-report scales to measure adherence with mixed results. ${ }^{11-14}$ These observed differences in agreement may be due to the dissimilar disease states and self-report scales examined in each study.

Within the past decade, the development of medication therapy management (MTM) services has created new opportunities for pharmacists. One of the 5 core elements of MTM service is medication therapy review, which includes an assessment of medication adherence. ${ }^{15}$ Rickles and Svarstad reported moderate to strong concordance between self-reported adherence from a modified BMQ and pharmacy refill records for antidepressant medication. ${ }^{14}$ The authors also note that through the use of simple selfreport questions, a pharmacist can detect adherence problems early, and provide appropriate interventions

to improve adherence. ${ }^{14}$ To our knowledge, no data exists on how well self-report tools correspond with a pharmacist's assessment of medication adherence based upon information reported during a medication therapy review. The goal of this paper will be to evaluate the concordance of patient's self-reported adherence using the 6-item Modified Morisky scale with a pharmacist's assessment of adherence during a medication review. Results of our study will be used to evaluate how a self-report scale may be used during a pharmacist-provided medication review.

\section{METHODS}

This retrospective, cross sectional study examined the electronic medical records of patients who received medication review by a pharmacist from October 2008 to September 2009. Eligibility criteria 
included: patients age 18 and older with a mental health and/or substance abuse diagnosis, received a least one medication review by a pharmacist in the mental health clinic at a health care for the homeless clinic, and had recorded responses to at least the first four questions (the original 4-item Morisky scale) of the 6-item Modified Morisky scale.

\section{Setting/Medication Review}

All medication reviews took place in a federally funded health care for the homeless clinic located in Richmond, Virginia. This health care clinic provides medical, dental, vision, mental-health, and case management services to homeless or patients at risk of experiencing homelessness. Since 2005, the health care clinic has had a community-academic partnership with the Virginia Commonwealth University School of Pharmacy. ${ }^{16}$ This partnership allows clinical faculty members of the school of pharmacy to provide medication therapy review services to patients of the medical and mental-health clinics. Medication review services are intended to assess the patient's medication adherence and provide education and/or interventions as necessary. ${ }^{16}$ Medication reviews begin with the pharmacist administering a brief self-report questionnaire and documenting the patient's answers in the electronic medical record. The pharmacist also inquires about the patient's use of OTC medications, herbal supplements, caffeine, tobacco, alcohol, and illicit drugs. Pharmacists attempt to identify barriers to adherence and examples of non-adherence by asking probing questions about medication taking behavior, having the patients demonstrate how they take the medication, and engaging the patient to discuss any difficulties they have related to the medication.

\section{Scales and Scoring}

The Case Management Society of America (CMSA) created a 6-item modified Morisky scale (MMS) which included the original four questions of the Morisky MAS 4-item scale, plus two additional questions to address persistence of taking medication. ${ }^{17}$ This 6-item questionnaire measures two domains of adherence; knowledge and motivation. ${ }^{17}$ The World Health Organization's 2003 report concerning medication adherence listed motivation and knowledge as patient-related factors affecting adherence. ${ }^{18}$ Patients are categorized as having "high" or "low" knowledge and motivation based on their yes/no response to each of the six questions of the MMS. ${ }^{17}$ Patients are then assigned to one of 4 adherenceintention quadrants based on their scores of motivation and knowledge. This quadrant can be used to identify deficiencies in knowledge and motivation and ultimately implement a plan to improve adherence.

${ }^{17}$ Methods to improve a patient's motivation and knowledge score include educating the patient on the purpose of the medication, the disease state being treated, adverse side effects to be aware of, and ensuring the patient understands how to take the medicine. The Case Management Adherence Guidelines (CMAG) list pharmacists as a resource to educate patients about their medication and to reinforce adherence. $^{17}$

At each medication review of our study, pharmacists administered and recorded patients' responses to the 6-item MMS. The use of the 6-item scale in this clinic was chosen over the 4-item MAS for its ability to assess long-term medication adherence and its ease of use during patient interview. Although the clinic in which this study took place does not use the 6-item scale to score adherence and classify patients based on motivation and knowledge domains, as does the CSMA, we included this classification in our analysis with pharmacist's assessment of adherence for completeness.

\section{6-item Scale (6-item MMS)}


The 6-item MMS contains six questions in which patients respond yes or no. Each "no" response to each question results in +1 (except question 5 , in which a "yes" response would result in +1 ) and "yes" responses to each question result in +0 (except question 5 , where a "no" response would result in +0 ). Scores for the 6-item MMS can range from 0 to 6, with higher scores indicating better adherence. The CMSA further created 2 domains of adherence (knowledge and motivation) measured by the 6-item scale. Questions 1,2, and 6 intend to assess a patient's motivation for medication adherence, while questions 3, 4 , and 5 reportedly assess a patient's knowledge of the medication. Measuring these 2 domains are partly influenced by the World Health Organization's 2003 report concerning medication adherence listed motivation and knowledge as patient-related factors affecting adherence. ${ }^{18}$ The motivation and knowledge domain scores are obtained from the 6-item scoring. Patients are considered to have "high" motivation or knowledge if they receive a score of $\geq 2$ for each domain; patients with scores of $\leq 1$ for each adherencedomain would be classified as "low" motivation or knowledge...

\section{5-item Scale}

The 5-item scale was developed from the 6-item scale by excluding question five, "Do you know the long-term benefits of taking your medication...?”. Initially, our analysis was to include only the 6-item scale, but in discussions with one of the pharmacists who conducted the medication reviews, we hypothesized that patients may not know the long-term benefits of the medication, but still be adherent by definition. The 5-item scale we created contained the same questions from the 6-item scale, excluding question 5. This scale was scored similar to the 6 -item, with each "no" response resulting in +1 , and "yes” responses +0 . Scores could range from 0 to 5, with higher scores indicating better adherence.

\section{4-itme Scale}

Previously, we detailed how the 5-item scale was created by excluding question 5 of the 6-item scale. Additionally, we thought to exclude question 6 of the 6-item scale, "Do you sometimes forget refills?" due to the unique characteristics of our study population. Patients at this health care clinic must present monthly to obtain refills for their medication. If patients were unable to attend an appointment (due to lack of transportation, incarceration, hospitalization, or any reason), they were unable to obtain medication refills. Patients with missed appointments were then considered to have "forgotten" refills, when in truth, they were unable to maintain their appointment. The 4-item scale used is the original Morisky MAS. ${ }^{6}$ The Morisky scale was first validated in assessing medication adherence in a population of predominantly African-American women with hypertension. ${ }^{6}$ The original scale classifies patient's adherence as "high, medium, or low" based on the number of "yes" responses to each of the 4 questions. Patients who answered, “yes” to 1 or 2 of the questions were identified as "medium adherence” and patients who had answered, "yes" to 3 or 4 questions were classified as "low adherence”. Our analysis scored the 4-item scale identically to the 5 -item and 6-item scales, by awarding +1 for each "no" answer, and +0 for each "yes" answer. Scores then ranged from 0 to 4, with higher scores indicating better adherence. We chose to analyze agreement between pharmacist assessment of adherence with the 4-item, 5-item, and 6-item scales and examine how each scale compared.

\section{Defining Non-Adherence}

During medication review, pharmacists administered the 6-item MMS and had the opportunity to ask the patient a series of questions regarding use of their medications. At the conclusion of the medication review, pharmacists created a problem list, which may have included medication-related problems such as 
ineffective dosing, duplication of medication therapy, and non-adherence. Patients in this study were categorized as "non-adherent" by pharmacist assessment if non-adherence was noted in the problem list generated at the conclusion of the medication review. All patients whose problem list did not include non-adherence were considered "adherent" in this study.

Patients were considered non-adherent for each self-report scale if they scored $\geq 1$. We chose to dichotomize adherence into adherent/non-adherent categories to match the "non adherent/adherent" classification used by pharmacist assessment. Additionally, a previous study had defined non-adherence measured by the 4-item Morisky in the same way, when comparing self-report to electronic monitoring (MEMS) data in patients taking tricyclic antidepressants. This group reported 84\% sensitivity and 55.4\% specificity when non-adherence was defined as a score of $\geq 1$ (a "yes" response to any question) on the 4item scale. $^{10}$

\section{ANAYLYSIS}

Statistical analysis was conducted using SAS software. Descriptive statistics were used for all sociodemographic and adherence variables. Kappa statistic with $95 \%$ confidence interval was used to examine the agreement between pharmacist assessments of non-adherence with non-adherence defined by self-report scales. Phi coefficient was used to examine the relationship between pharmacist assessment of adherence and dichotomous levels of motivation/knowledge measured by the 6-item scale.

\section{RESULTS}

A total of 288 patients were eligible for this study, which included 449 medication reviews from October 2008 to September 2009. The sample was 51.7\% female, 60.4\% African American, and had a mean age of $44.8 \pm 10.1$ years. The mean number of mental health conditions was $2.3 \pm 0.9$ and the mean number of medications at baseline was $3.5 \pm 2.4$. Nearly one-half of patients (45.8\%) reported to being homeless or doubling up with family or friends. The number of medication reviews ranged from 1 to 8 , with a majority of patients (59\%) receiving 2 or more medication reviews with a pharmacist. The percentage of patients identified as non-adherent by each method of assessment are shown in table 1. Non-adherence determined by pharmacist assessment was determined in nearly $24 \%$ of medication reviews. Non-adherence determined by self-report scale seemed to increase as the number of self-report questions increased. Table 2 shows data for non-adherence assessed by each method. Agreement between pharmacist assessment of non-adherence and non-adherence by each self-report scale is reported in table 3. Agreement was highest between pharmacist assessment and non-adherence determined by the 4-item Morisky scale, but showed only a fair correlation. Agreement between pharmacist assessment and 6 -item scale showed the lowest agreement with a kappa value of 0.17 , indicating weak agreement. Although the 6-item scale is not used as described by the CMAG in our study population, we examined the relationship between pharmacist's assessment of adherence and levels of motivation/knowledge, presented in table 4. Levels of both knowledge and motivation were found to be weakly related to pharmacist assessment of adherence. 


\section{DISCUSSION}

This study examined the concordance of medication non-adherence assessed by a licensed pharmacist with non-adherence detected by Morisky self-report scores. We found that the 4-item Morisky, 5-item scale, and 6-item Modified Morisky, had fair to weak correlation with pharmacist assessment of medication non-adherence. Agreement between pharmacist assessment and self-report scale for determining non-adherence seemed to decrease as the number of self-report questions increased. Regardless of the number of questions, self-report scales used in this study did not show high agreement with pharmacist assessment. Our results show that pharmacist assessment detected the lowest proportion of non-adherence (23.83\%), while the 6-item scale predicted the highest proportion of non-adherence (61.66\%). Interestingly, the proportion of medication reviews determined non-adherent by 4-item Morisky scale (49.67\%) falls within the estimated value of non-adherence mentioned in the literature. ${ }^{1}$

The low non-adherence detected by pharmacists may be due to variation in pharmacist assessment. Three different pharmacists conducted the medication reviews during the time period of this study. In addition, each pharmacist may have had a different method of conducting medication review and assessing non-adherence. Likewise, each pharmacist's definition of non-adherence and/or the criteria by which they classified non-adherence is likely to vary. It is also important to point out the criteria in which we determined non-adherence assessed by pharmacist. Only patients with medication non-adherence listed in the medication problem list were categorized as non-adherent in this study. We believe this limitation, along with the variability in pharmacist assessment, may have lead to an underestimate of nonadherence when assessed by pharmacist. We also acknowledge several limitations that come from the use of self-report scales to measure non-adherence. First, although the 4-item Morisky scale has been validated in assessing medication adherence in several disease states, it has not been validated in assessing medication adherence in mental health disorders. Previously it was mentioned that the 4-item had been validated in assessing adherence to tricyclic antidepressants. ${ }^{10}$ It should be noted that TCAs have largely been replaced by newer classes of antidepressants (SSRI/SNRI) to treat depression. Use of the 6-item scale has limited data to suggest that it is validated in assessing medication adherence with any class of medications.

An additional limitation of using any self-report scale is the reliability of patient's response. Several studies have shown that self-reported adherence is often overestimated.,11-13 Our conservative definition of non-adherence may be an additional limitation, and may have resulted in an overestimation of nonadherence assessed by self-report. George et al. (2000) reported a high sensitivity (84\%) in dichotomizing adherence by 4-item Morisky score (adherent $=0$; non-adherent $=\geq 1$ ) compared with adherence measured by electronic monitoring (MEMS) data (adherence $=100 \%$ compliant; nonadherence $=<100 \%$ compliant). When the threshold for adherence, measured by MEMS data, decreased to $90 \%$, the calculated sensitivity also decreased to $75 \% .{ }^{10}$ If we modify our definition of non-adherence to answering “yes” to 2 or more questions on any self-report scale, we expect the proportion of medication reviews determined non-adherent to decrease, with an expected decrease in sensitivity. As a result, better agreement between pharmacist assessment and self-report may be observed, but with less confidence in detecting true non-adherence. Modifying the definition of adherence to include patients who are partially adherent, distorts the definition of adherence. Although each question of the self-report questionnaire measures a certain aspect of adherence, each question of the self-report may not be equally important at assessing adherence. Additionally, it is difficult to quantify a percentage of medication 
adherence that serves as a threshold for optimal outcomes. These limitations make the use of self-report scales to difficult to incorporate into clinical practice.

Lastly, the use of the Morisky scale, or any self-report scale, in patients with mental-health disorders who are homeless or at risk of homelessness may be invalid and/or unreliable. Patients with psychiatric disorders are at an increased risk of non-adherence due to lack of insight, psychotic symptoms, and low social functioning $^{16}$. Additionally, psychiatric disease is highly prevalent among homeless persons, who are at an increased risk for non-adherence due to high incidences of substance abuse, illiteracy, and lack of health care access. ${ }^{16}$ These risk factors should be considered when choosing a method for assessing medication adherence in this unique population.

When patients were categorized into levels of motivation and knowledge based on scores to the 6-item MMS, a patient's level of knowledge or motivation showed a weak relationship to pharmacist assessment of adherence. Although pharmacists in this study do not use the 6-item MMS to classify patients based on knowledge and motivation, as described by the Case Management Adherence Guidelines, we included this measure in our analysis. The 6-item scale was chosen for its ability to be quickly administered during interview and its assessment of long-term benefits. Pharmacists desired a scale that could assess a patients knowledge regarding the long-term benefits of medication adherence, as certain mental-health medications may take up to 6 to 12 weeks to show effectiveness. The 6-item Modified Morisky scale, as used in this study, may not be appropriate for simply identifying non-adherent patients served at a health care for the homeless clinic.

The results of this study have several implications for improving the delivery of medication therapy review by pharmacists. First, when multiple pharmacists are conducting medication reviews, a standardized method for review may help eliminate variation in assessing adherence. Providing an initial training on how medication reviews should be conducted may help improve the consistency by which non-adherence is determined. One pharmacist in our study stated that they used the self-report questionnaire as a way to follow up on areas of non-adherence highlighted by patient response to the selfreport questions. Also, it is imperative that all pharmacists involved in the medication review process have a clear and unanimous definition of non-adherence. Perhaps most important is determining which, if any, self-report questionnaire should be used. If a self-report questionnaire will be used, pharmacists should agree upon which self-report to use and what its purpose will be. Lastly, if non-adherence is to be measured by pharmacist's assessment, it may be beneficial to include a dedicated area in the medical record to categorize the patient as adherent or not. A simple box indicating adherent or non-adherent would eliminate any ambiguity of having to interpret what was written in the note. Additionally, including a free text area to specify what type of adherence barriers are suspected would be useful to other clinicians when evaluating the patient at future visits.

Our study showed weak agreement between Morisky self-reports and pharmacist assessment. Better agreement between pharmacist assessment of adherence may be seen if other indirect measures (pharmacy refill data) are used in addition to self-reported adherence. Evaluating medication adherence through indirect measures such as pharmacy refill data may not be appropriate or possible in this population due to patients using multiple pharmacies, or obtaining medications in the form of samples from prescribers. Self-report measures to detect non-adherence, by 3 versions of the Morisky scale, did not show strong agreement with non-adherence assessed by a pharmacist during medication review. Also, categories of high and low motivation/knowledge from the 6-item MMS were weakly related to 
pharmacist assessment. Self-report measures of adherence are considered by some to be inferior to other indirect measures because of patient overestimates of adherence. However, in some circumstances, selfreport questionnaires may be useful to pharmacists as a fast, and inexpensive way to identify patients with multiple barriers to adherence. Self-report scales may also be a useful way of engaging the patient to discuss any adherence problems and allow the pharmacist to provide appropriate education and interventions.

\section{REFERENCE}


1. Osterberg L, Blaschke T. Adherence to medication. $N$ Engl J Med. 2005; 353(5): 487497.

2. NEHI. (2009). Thinking outside the Pillbox: A System-wide Approach to Improving Patient Medication Adherence for Chronic Disease. Cambridge, MA. New England Healthcare Institute.

3. NEHI. (2012). Thinking outside the Pillbox: Improving Medication Adherence and Reducing Readmissions. Cambridge, MA. New England Healthcare Institute.

4. Fairman, K., \& Motheral, B. (2000). Evaluating Medication Adherence: Which Measure is Right for Your Program? Journal of Managed Care, 6, 499-504.

5. Lavsa SM, Holzworth A, Ansani NT. Selection of a validated scale for measuring medication adherence. J Am Pharm Assoc (2003). 2011;51(1):90-94.

6. Morisky DE, Green LW, Levine DM. Concurrent and predictive validity of a selfreported measure of medication adherence. Med Care. 1986; 24(1):pp. 67-74.

7. Morisky DE, Ang A, Krousel-Wood M, Ward HJ. Predictive validity of a medication adherence measure in an outpatient setting. J Clin Hypertens. 2008;10(5):348-354.

8. Shalansky SJ, Levy AR, Ignaszewski AP. Self-reported Morisky score for identifying nonadherence with cardiovascular medications. Ann Pharmacother. 2004;38(9):13631368.

9. Krousel-Wood M, Islam T, Webber LS, Re RN, Morisky DE, Muntner P. New medication adherence scale versus pharmacy fill rates in seniors with hypertension. Am J Manag Care. 2009;15(1):59-66.

10. George CF, Peveler RC, Heiliger S, Thompson C. Compliance with tricyclic antidepressants: The value of four different methods of assessment. Br J Clin Pharmacol. 2000;50(2):166-171.

11. Guenette L, Moisan J, Preville M, Boyer R. Measures of adherence based on self-report exhibited poor agreement with those based on pharmacy records. J Clin Epidemiol. 2005;58(9):924-933.

12. Wang PS, Benner JS, Glynn RJ, Winkelmayer WC, Mogun H, Avorn J. How well do patients report noncompliance with antihypertensive medications?: A comparison of selfreport versus filled prescriptions. Pharmacoepidemiol. Drug Safety. 2004; 13 (1) 11-19.

13. Velligan DI, Wang M, Diamond P, et al. Relationships among subjective and objective measures of adherence to oral antipsychotic medications. Psychiatr Serv. 2007;58(9):1187-1192.

14. Rickles NM, Svarstad BL. Relationships between multiple self-reported nonadherence measures and pharmacy records. Res Social Adm Pharm. 2007;3(4):363-377.

15. American Pharmacists Association, National Association of Chain Drug Stores Foundation. Medication therapy management in pharmacy practice: Core elements of an MTM service model (version 2.0). J Am Pharm Assoc (2003). 2008;48(3):341-353.

16. Gatewood SB, Moczygemba LR, Alexander AJ, et al. Development and implementation of an academic community partnership to enhance care among homeless persons. Inov Pharm. 2011;2(1):1-7. 
17. Case Management Society of America. (2006). Case Management Adherence Guidelines: Version 2.0. Little Rock, AR.

18. World Health Organization. (2003). Adherence to Long-Term Therapies - Evidence for Action. Geneva 27, Switzerland. World Health Organization.

Table 1: Number of medication review sessions identified as adherent or non-adherent from self-report scores compared to pharmacist assessment.

Pharmacist Assessment

Adherent $\quad$ Non-Adherent




\begin{tabular}{|c|c|c|c|c|}
\hline 4-item & Adherent & 198 & 28 & $\mathrm{~N}=226$ \\
\hline \multirow{2}{*}{\multicolumn{2}{|c|}{ Non-Adherent }} & 144 & 79 & $\mathrm{~N}=223$ \\
\hline & & $\mathrm{N}=342$ & $\mathrm{~N}=107$ & \\
\hline 5-item & Adherent & 177 & 24 & $N=201$ \\
\hline \multirow{2}{*}{\multicolumn{2}{|c|}{ Non-Adherent }} & 162 & 83 & $\mathrm{~N}=245$ \\
\hline & & $\mathrm{N}=339$ & $\mathrm{~N}=107$ & \\
\hline 6-item & Adherent & 151 & 20 & $\mathrm{~N}=171$ \\
\hline \multirow{2}{*}{\multicolumn{2}{|c|}{ Non-adherent }} & 188 & 87 & $\mathrm{~N}=275$ \\
\hline & & $\mathrm{N}=339$ & $\mathrm{~N}=107$ & \\
\hline
\end{tabular}

Table 2: Non-Adherence rates as determined by method of assessment.

\begin{tabular}{|c|c|c|}
\hline Non-Adherence (\%) & Method of Detection & N \\
\hline 23.83 & Pharmacist Assessment & 449 \\
\hline 49.67 & 4-item Self-Report & 449 \\
\hline 54.93 & 5-item Self-Report & 446 \\
\hline 61.66 & 6-item Self-Report & 446 \\
\hline *Differences in number of medication reviews for analysis of the 5-item and 6- \\
\hline
\end{tabular}

Table 3: Correlation between pharmacist assessment of adherence and 4-item, 5-item, and 6-item scales.

\begin{tabular}{|c|c|c|c|}
\hline \multicolumn{2}{|c|}{ Method of Comparison } & N & Phi coefficient \\
\hline Pharmacists assessment & Motivation & 446 & 0.19 \\
\hline Pharmacists assessment & Knowledge & 449 & 0.20 \\
\hline
\end{tabular}

Table 4: Relationship between pharmacist assessment of adherence and patient level of motivation and knowledge.

\begin{tabular}{|c|c|c|c|}
\hline \multicolumn{2}{|c|}{ Method of Comparison } & N & Kappa statistic \\
\hline Pharmacist assessment & 4-item Morisky & 449 & 0.23 \\
\hline Pharmacist assessment & 5-item & 446 & 0.21 \\
\hline Pharmacist assessment & 6-item & 446 & 0.17 \\
\hline
\end{tabular}

